\title{
The yellow and the black of synthetic melanins
}

Koen P. Vercruysse and Venise Govan

Chemistry Department, Tennessee State University, Nashville, USA

Corresponding author: kvercruysse@tnstate.edu

\begin{abstract}
The observations reported here are a continuation and expansion of observations described earlier: the typical conditions to synthesize melanin-like materials from catecholic precursors (air-oxidation in an alkaline environment) leads to the generation of a light-colored substance in addition to the typical dark substances. This light-colored substance appears to be associated with the dark-colored materials through non-covalent interactions. We employed a novel co-precipitation process that allows for a simple separation of this light-colored substance from the dark-colored materials. We studied some of the physic-chemical properties (color, fluorescence, FT-IR absorbance) of some of the fractions we obtained and discussed these properties in the context of what has been discussed for eumelanin, pheomelanin or neuromelanin. Overall, the current observations strengthen our hypothesis that synthetic melanins may be built from at least two types of substances: 1) a soluble, yellow-to-orange colored component, possibly rich in unoxidized precursors and 2) an insoluble, dark component. Our observations do suggest that when studying the chemistry or physiology of melanins one should look beyond the dark colors typically displayed by melanin-like materials.
\end{abstract}

Keywords: melanin, catechol, pyrogallol, dopamine, L-DOPA, spectroscopy

\section{Introduction}

Building upon earlier observations and the hypotheses derived from them, we explored further the possibility that melanins (MNs), synthesized through air-oxidation in an alkaline environment, consist of at least two distinctly different types of materials. ${ }^{1-3}$ We observed that from within the dark-brown to black material typically generated during the MN synthesis process, one can extract substances that possess a much lighter (orange to yellow) color. We have expanded these observations in part with the inclusion of catechol and pyrogallol as precursors for MN-like materials. The chemical structures (see Figure 1) of these two precursors are much simpler compared to L-DOPA or dopamine and it was hoped that the use of catechol or pyrogallol would lead to easier-to-characterize MN-like materials. 


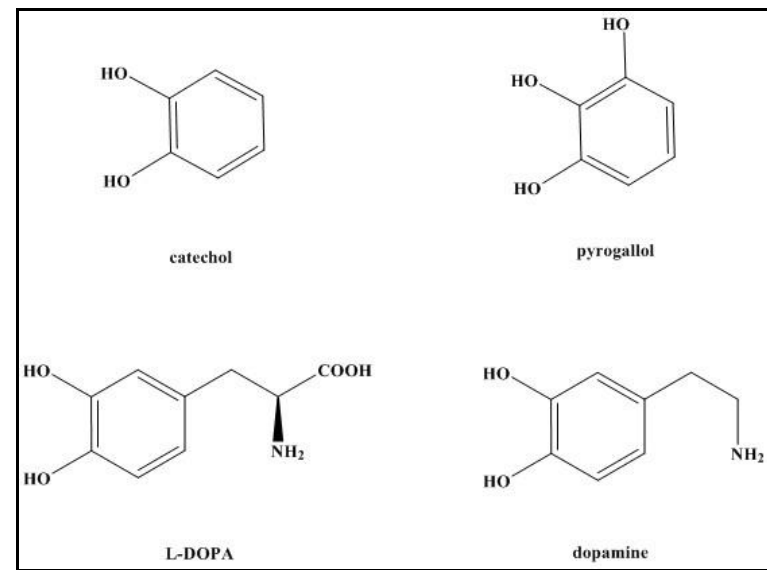

Figure 1: Chemical structures of the melanin precursors used in this study.

MNs are present in all kingdoms of life and their importance in biochemistry or physiology is well-recognized. ${ }^{4-7}$ Despite decades of extensive research, MNs remain one of the most enigmatic classes of biomolecules that still await full chemical and physical characterization. Consequently, the literature does contain discussions as to what constitutes "melanin". Broad definitions of MNs have been suggested: "MNs are heterogeneous polymers derived by the oxidation of phenols and subsequent polymerization of intermediate phenols and their resulting quinones"4, or "MNs are pigments of diverse structure and origin derived by the oxidation and polymerization of tyrosine in animals or phenolic compounds in lower organisms" ${ }^{8}$. Thus, different types of $\mathrm{MN}$-like materials exist depending on the precursor compound involved. ${ }^{9}$

In human physiology, MNs are present in skin, eyes, brain and other tissues or organs. ${ }^{5}$ The MNs present in the skin or hair are subdivided into eumelanin and pheomelanin. Eumelanin exhibits a brown to black appearance, while pheomelanin exhibits a yellow to red appearance. ${ }^{10}$ Information regarding the chemical features of MNs has been obtained through degradation studies of natural or synthetic MNs. In this context, the Raper-Mason scheme has long been accepted as the foundation to describe the initial stages of the melanogenesis process. In this scheme, tyrosine is oxidized to L-DOPA and converted into subsequent intermediates like dopaquinone, cyclodopa, 5,6-dihydroxyindole (DHI) and 5,6-dihydroxyindole-2-carboxylic acid (DHICA), which then form the final eumelanin material. ${ }^{10}$ However, despite its long-standing reputation, the validity of the Raper-Mason scheme has been called into question. ${ }^{11}$ Similarly, the synthesis of pheomelanin has been described as the result of the formation of adducts between Lcysteine and oxidation products of L-DOPA leading to intermediates like 5-S- or 2-S- 
cysteinyldopa and 1,4-benzothiazine compounds. ${ }^{10}$ In comparison to eumelanin, much less literature exists on the structure and properties of pheomelanin. ${ }^{10,12}$

The $\mathrm{MN}$ in the brain is referred to as neuromelanin and is described as a reaction product obtained through the oxidation of dopamine in the presence or absence of L-cysteine. ${ }^{13}$ In other organisms, particularly in plants, bacterial or fungal species, nitrogen-free precursors, e.g., catechol, pyrogallol, homogentisic acid or dihydroxynaphtalene, form the basis of the MN-like materials these organisms generate. ${ }^{4}$

MNs are typically described as dark substances, insoluble in water or any type of organic solvent and with no fluorescent emission. However, throughout the literature there are reports of MN-like materials that appear soluble in water ${ }^{14-17}$, that appear lighter in color ${ }^{14,18-19}$ or exhibit fluorescent properties. ${ }^{14,20-25}$ Most MNs exhibit dark colors and are capable of absorbing energy over the UV, visible and near-infrared range of the electromagnetic spectrum; typically exhibiting a monotonic decline in absorbance with increasing wavelength. ${ }^{9,}{ }^{26}$ Although the chemical structure of any of the MNs has not been established, there appears to be a consensus that MNs are "chemically disordered", i.e., built from a mixture of chemical entities. ${ }^{27}$ In this "chemical disorder model", the superimposition of the absorption profiles of the individual components would lead to the overall, featureless, broad and exponentially declining UV-Vis absorbance profile. ${ }^{24,27-28}$ However, the "chemical disorder" model to explain the monotonic UV-Vis absorbance profile has been criticized and physical effects, e.g., stacking of oligomers, has been emphasized as a possible explanation for the materials' absorption profile. ${ }^{29-30}$

In terms of physical arrangement, there appears to be a consensus that MNs built from LDOPA or dopamine are organized into multilayered entities through non-covalent interactions. Washing with alkaline solutions has been applied to disassemble such materials into various subfractions. $^{20,31-35}$

Synthetic MNs are investigated for a multitude of possible applications, particularly for their adhesive properties as commonly studied for polydopamine (PDA) which is another term used for MN-like materials derived from dopamine. ${ }^{19,21,36}$ The reader is referred to recent reviews regarding the current trends in the application of MN-based materials. ${ }^{28,37-38}$ In order to relate functionality (application) to chemical structure, any insights in the physic-chemical properties of the reaction products generated through the typical synthetic melanogenesis process is of importance. 


\section{Materials and Methods}

\subsection{Materials}

Catechol, pyrogallol, dopamine.HCl, L-DOPA and all other chemicals were obtained through Fisher Scientific (Suwanee, GA, USA).

\subsection{UV-Vis analyses and evaluation of the "darkness" of a sample}

UV-Vis spectroscopy was performed inside microwells of 96-well microplates using the SynergyHT microplate reader from Biotek (Winooski, VT). $200 \mu \mathrm{L}$ aliquots of samples, diluted whenever needed, were used and $200 \mu \mathrm{L}$ water as the blank. UV-transparent 96-well microplates were used for the evaluation of the absorbance of any sample within the UV range of the electromagnetic spectrum. All measurements were performed at room temperature.

The "darkness" of any sample was evaluated using a process outlined elsewhere. ${ }^{1}$ Absorbance spectra are recorded between 350 and 900nm. The absorbance between 500 and $800 \mathrm{~nm}(700 \mathrm{~nm}$ for particularly light-colored samples) is fitted with an exponential equation. The corresponding equation is integrated between 400 and $900 \mathrm{~nm}$; essentially calculating the area-under-the-curve (AUC) of the absorbance profile within the visible range of the spectrum as shown in equations 1 and 2.

$$
\begin{aligned}
& A=A_{0} * e^{-k \lambda} \\
& A U C=\int_{400}^{900}\left(A_{0} * e^{-k \lambda} d \lambda\right)=\frac{A_{0}}{-k} *\left(e^{-k * 900}-e^{-k * 400}\right)
\end{aligned}
$$

In these equations, $\lambda$ is the wavelength, $\mathrm{A}$ is the measured absorbance at a particular $\lambda, \mathrm{A}_{0}$ is the absorbance of the sample at $\lambda=0$ and $\mathrm{k}$ is a decay constant.

\subsection{Fluorescence spectroscopy}

Fluorescence measurements were made in wells of an opaque 96-well microplate using the SynergyHT microplate reader from Biotek (Winooski, VT) with excitation filter set at 360nm, emission set at $460 \mathrm{~nm}$ and sensitivity factor set at 75 .

\subsection{Size-exclusion chromatography (SEC) analyses}

SEC analyses were performed on a Breeze 2 HPLC system equipped with two 1500 series HPLC pumps and a model 2998 Photodiode array detector from Waters, Co (Milford, MA). Analyses were performed using an Ultrahydrogel 500 column (300 X $7.8 \mathrm{~mm}$ ) obtained from 
Waters, Co (Milford, MA) in isocratic fashion using a mixture of $25 \mathrm{mM} \mathrm{Na}-$ acetate:methanol:acetic acid (90:10:0.05\% v/v) as solvent.

\subsection{Dialysis and lyophilization}

Dialysis was performed using Spectrum Spectra/Por RC dialysis membranes with molecularweight-cut-off of 3.5kDa obtained from Fisher Scientific (Suwanee, GA, USA) against water (up to $3.5 \mathrm{~L}$ ) for two days with three changes of water during that time. The dialyzed mixtures were kept at $-20^{\circ} \mathrm{C}$ for 24 hours and dried for three days using a Labconco FreeZone Plus 4.5L benchtop freeze-dry system obtained from Fisher Scientific (Suwanee, GA, USA)

\subsection{FT-IR spectroscopy}

FT-IR analyses were performed using the Spectrum Two FT-IR spectrometer from PerkinElmer (Waltham, Massachusetts). Scans were made using the universal ATR accessory between 650 and $4,000 \mathrm{~cm}^{-1}$ with a resolution of $4 \mathrm{~cm}^{-1}$ and using the OptKBr beam splitter and $\mathrm{LiTaO}_{3}$ detector. For each sample 24 scans were accumulated.

\subsection{Synthesis and fractionation of melanins - part 1}

About 300mg precursor was dissolved in 50mL acetate buffer $(50 \mathrm{mM}$; pH=6.5) and the reaction was initiated by the addition of $50 \mathrm{~mL} \mathrm{Na} \mathrm{CO}_{3}$ at $50 \mathrm{mM}$. In the case of dopamine. $\mathrm{HCl}$, $25 \mathrm{~mL} 50 \mathrm{mM}$ acetate buffer and $75 \mathrm{~mL} 50 \mathrm{mM} \mathrm{Na} \mathrm{CO}_{3}$ were used to compensate for the acidity of the hydrochloride form of dopamine. The mixtures were left stirring at room temperature and monitored using SEC to ensure that the precursor compound had reacted away.

To evaluate the effect of the co-precipitation with $\mathrm{CaCO}_{3}, 500 \mu \mathrm{L}$ aliquots from the crude reaction mixtures were removed and mixed with $50 \mu \mathrm{L}$ water or $50 \mu \mathrm{L} \mathrm{CaCl}_{2}$ at concentrations ranging between 0.05 and 1.0M. After overnight standing and centrifugation, the "darkness" of the supernatants was evaluated as outlined earlier.

The work-up process applied to all reaction mixtures is outlined in Scheme 1. Each reaction mixture was divided into $20 \mathrm{~mL}$ fractions and to each fraction $2 \mathrm{~mL}$ of $0.5 \mathrm{M} \mathrm{CaCl}_{2}$ was added. These mixtures were left standing at room temperature overnight to let the precipitations settle. After centrifugation, the supernatants (fraction I) were pooled and the precipitate further washed with: a) $2 \mathrm{X} 10 \mathrm{~mL}$ water (kept separate as fractions II and III), b) $2 \mathrm{X} 10 \mathrm{~mL} 0.1 \mathrm{~N} \mathrm{HCl}$ (pooled into fraction IV) and c) 2 X 10mL water (pooled into fraction V). Select fractions thus obtained from each reaction mixture were dialyzed against distilled water, frozen and lyophilized. Any 
remaining precipitate was frozen and lyophilized. For all four precursors the reactions were set up and processed in duplicate.

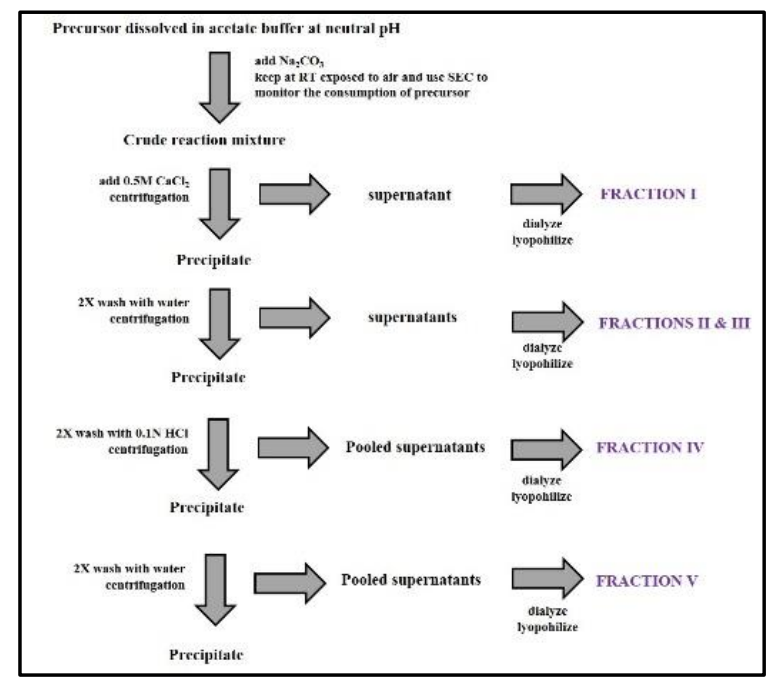

Scheme 1: Fractionation process of the synthetic MN-like materials.

\subsection{Synthesis and fractionation of melanins - part 2}

The fractionation process described above was studied in more detail using L-DOPA and dopamine. Reaction mixtures were set up containing 100mg L-DOPA or dopamine.HCl in a 30mL mixture of 50mM Na-acetate buffer:50mM Na $\mathrm{CO}_{3}$ at 15:15 (v/v) for L-DOPA or at 10:20 $(\mathrm{v} / \mathrm{v})$ for dopamine. The mixtures were left stirring at room temperature till all precursor materials were deemed reacted away as judged from SEC analyses. Two 10mL fractions from each mixture were mixed with $1 \mathrm{~mL} 0.5 \mathrm{M} \mathrm{CaCl}_{2}$ and left standing at room temperature to let precipitations settle. Upon centrifugation, the precipitates were: a) washed $10 \mathrm{X}$ with $5 \mathrm{~mL}$ distilled water or b) washed $2 \mathrm{X}$ with $5 \mathrm{~mL}$ distilled water, $3 \mathrm{X}$ with $5 \mathrm{~mL} 0.1 \mathrm{~N} \mathrm{HCl}$ and $5 \mathrm{X}$ with $5 \mathrm{~mL}$ distilled water. At every stage, aliquots from the supernatants were diluted whenever needed and their spectra in the visible (350 to 900nm) and UV-Visible (230 to 900nm) range were recorded. Any remaining precipitate was frozen and lyophilized. The remaining, untreated portions of the crude reaction mixtures were dialyzed against distilled water, frozen and lyophilized as outlined above. 


\section{Results}

\subsection{Evaluation of the co-precipitation with $\mathrm{CaCO}_{3}$}

Figure 2 compares, for all four precursors involved and as a function of $\mathrm{CaCl}_{2}$ used, the decline in relative darkness (based upon calculated AUC values as outline in Materials and Methods) of the supernatants obtained from the crude reaction mixtures upon co-precipitation of the MN-like material on $\mathrm{CaCO}_{3}$.

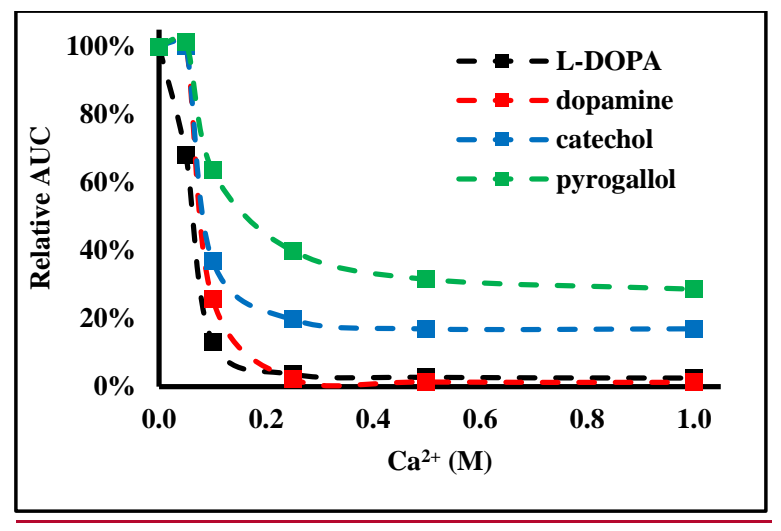

Figure 2: Relative darkness of the supernatants of reaction mixtures following co-precipitation of the MN-like materials on $\mathrm{CaCO}_{3}$ as a function of the $\mathrm{Ca}^{2+}$ concentration used to initiate the co-precipitation. The darkness, expressed as the relative change in AUC, was calculated as outlined in Materials and Methods.

The results presented in Figure 2 indicate that the dark materials generated from L-DOPA or dopamine can be co-precipitated on $\mathrm{CaCO}_{3}$ to a near $100 \%$ level, while 20 or $40 \%$ of the dark material generated from catechol or pyrogallol respectively remained in the supernatant solution at the highest levels of $\mathrm{Ca}^{2+}$ used. From this experiment it appeared that at a concentration of $0.5 \mathrm{M} \mathrm{CaCl}_{2}$, a maximum amount of dark material had co-precipitated. This level of $\mathrm{CaCl}_{2}$ was subsequently used for all further experiments.

\subsection{Observations during the work-up of the reaction mixtures.}

The series of photographs shown in Figure 3, panels A through E, illustrate the appearance of the various fractions obtained following the processing of the reaction mixtures involving catechol as the precursor. The series of photographs shown in Figure 3, panels F through J, illustrate the appearance of some select other fractions obtained during the experiments described in this report. 

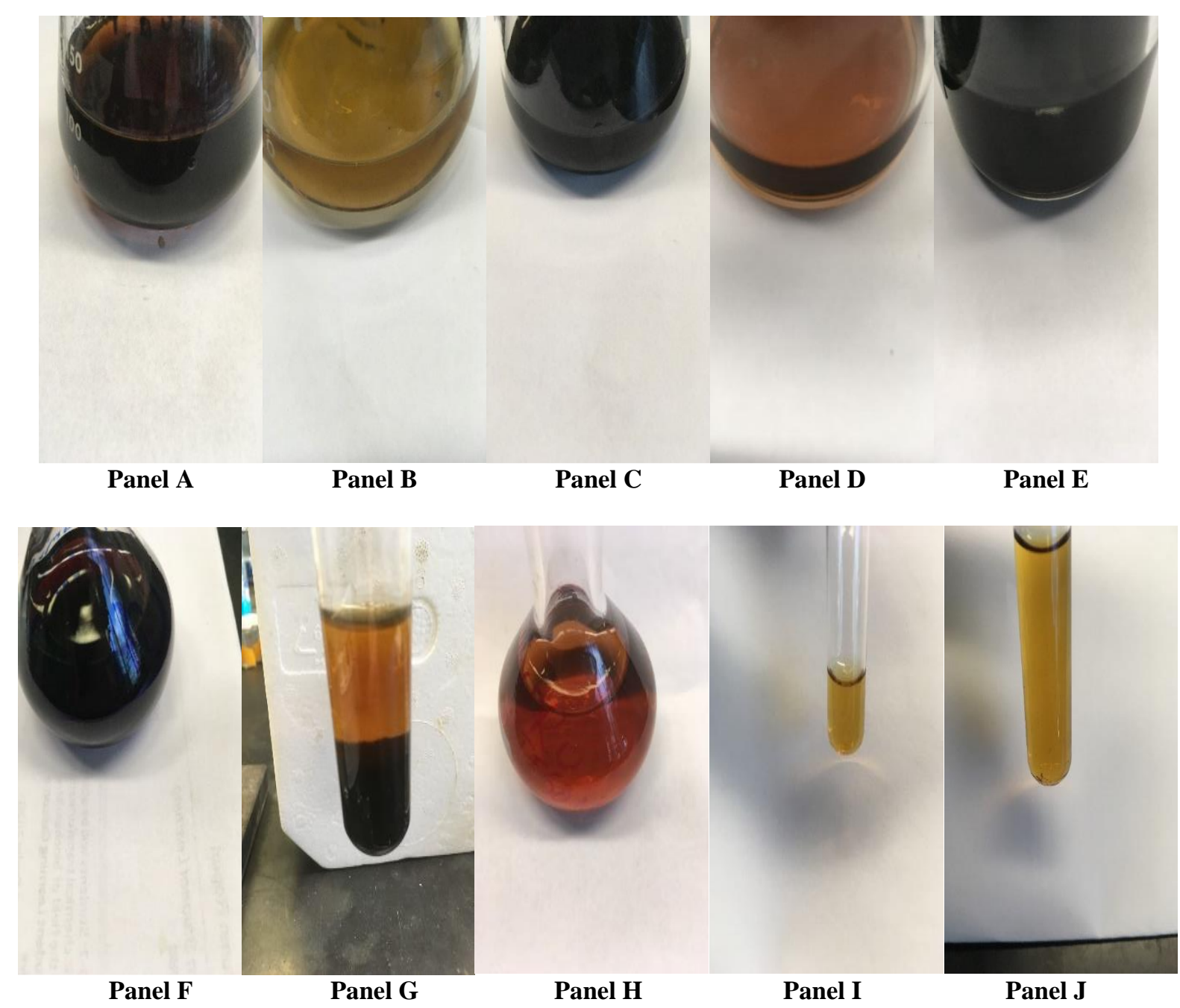

Figure 3: Photographs of fractions obtained during the work-up of the reaction mixtures. Panel A through E: Crude fractions I, II, III, IV and V respectively obtained from catechol. Panel F: Crude fraction IV obtained from pyrogallol. Panel G: Crude reaction mixture with dark precipitate and orange-colored supernatant obtained from LDOPA. Panel H: Crude pooled fraction I obtained from L-DOPA. Panels I and J: Purified fraction I obtained from L-DOPA and dopamine respectively dissolved in water at $1 \mathrm{mg} / \mathrm{mL}$.

For all crude reaction mixtures, the addition of $0.5 \mathrm{M} \mathrm{CaCl}_{2}$ resulted in a separation between a black precipitate and an orange-red supernatant (fraction I; for an example see Figure 3, panel G). In the cases of catechol and pyrogallol fraction I (see Figure 3, panel A) was much darker in appearance compared to the supernatants obtained for the reactions involving L-DOPA or dopamine. This observation is in accordance with the results presented in Figure 2.

For all precursors involved, fraction I would show signs of "physical instability", i.e., the formation of a black precipitate, upon standing at room temperature or during the dialysis process. This precipitate obtained from fractions I was not recovered and was not a part of the dried and purified fractions I. Figure 3, panels I and J, present photographs of solutions 
containing $1 \mathrm{mg} / \mathrm{mL}$ of purified Fraction I obtained from L-DOPA and dopamine, showing that these components exhibit a yellow color.

For all reaction mixtures, the first wash with water (fraction II; for an example see Figure 3, panel B) yielded a light-colored solution with minimal absorbance between 230 and 900nm. In all cases, fraction II was not processed further.

For all reaction mixtures, the second wash with water (fraction III; for an example see Figure 3, panel C) yielded a dark-colored solution which was dialyzed and lyophilized. As for fraction I, fraction III would show signs of "physical instability", upon standing at room temperature or during the dialysis process.

With the notable exception of the reactions involving pyrogallol, both washes with $0.1 \mathrm{~N} \mathrm{HCl}$ (fraction IV; see Figure 3, panels D and F) yielded light-colored solutions with minimal absorbance between 230 and 900nm. Only for the reactions involving pyrogallol, and for one reaction involving L-DOPA, was fraction IV dialyzed and lyophilized.

The subsequent washes with water (fraction V; for an example see Figure 3, panel E) yielded black-colored solutions for the reactions involving L-DOPA and catechol. These fractions were dialyzed and lyophilized. Fractions V for the reactions involving dopamine or pyrogallol were light-colored with minimal absorbance between 230 and 900nm. These fractions were not processed further.

Only in the cases of L-DOPA and dopamine did significant amounts of precipitate remain following all the washes and these precipitates were lyophilized.

For various fractions the lyophilization process yielded a combination of powdery material and "sticky" material, i.e., pigment stuck to the sides of the plastic tubes used for the drying process. Most often these "sticky" portions could be washed off the sides of the plastic tubes with water and dried anew into a powdery material. An exception to this was the drying of the one fraction IV obtained from a reaction with L-DOPA. This subfraction remained "sticky" even after lyophilizing it twice. In general, FT-IR analyses showed that, for any given fraction, there was no difference between the powdery portion and the "sticky" subfractions.

All reactions were performed in duplicate and although there were quantitative differences, from a qualitative point of view (UV-Vis and FT-IR spectra), the observations and results were reproducible. Table 1 presents typical yields of the various fractions obtained from the four different precursors. 
Table 1: Overview of typical yields obtained for the various fractions following the oxidation and subsequent fractionation of about $300 \mathrm{mg}$ catecholic precursor.

\begin{tabular}{|c|c|c|c|c|}
\hline & catechol & pyrogallol & L-DOPA & dopamine \\
\hline Fraction I & $71 \mathrm{mg}$ & $83 \mathrm{mg}$ & $36 \mathrm{mg}$ & $23 \mathrm{mg}$ \\
\hline Fraction III & $39 \mathrm{mg}$ & $33 \mathrm{mg}$ & $14 \mathrm{mg}$ & $92 \mathrm{mg}$ \\
\hline Fraction IV & $\mathrm{n} / \mathrm{d}$ & $39 \mathrm{mg}$ & $\mathrm{n} / \mathrm{d}$ & $\mathrm{n} / \mathrm{d}$ \\
\hline Fraction V & $18 \mathrm{mg}$ & $\mathrm{n} / \mathrm{d}$ & $50 \mathrm{mg}$ & $\mathrm{n} / \mathrm{d}$ \\
\hline $\begin{array}{l}\text { Remaining } \\
\text { precipitate }\end{array}$ & $\mathrm{n} / \mathrm{d}$ & $\mathrm{n} / \mathrm{d}$ & $60 \mathrm{mg}$ & $61 \mathrm{mg}$ \\
\hline
\end{tabular}

\subsection{UV-Vis spectroscopic analyses}

All dialyzed and lyophilized fractions were dissolved or dispersed in distilled water. For many fractions, the purified material would not dissolve entirely and the UV-Vis absorbance of their supernatant (at an unknown concentration) was measured. For the soluble materials, their UVVis absorbance was measured at a concentration of 0.1 or $0.25 \mathrm{mg} / \mathrm{mL}$ depending on the intensity of their colors. Figure 4, panels A through D, presents the results thus obtained and for comparison purposes all spectra were normalized for their absorbance at 280nm.

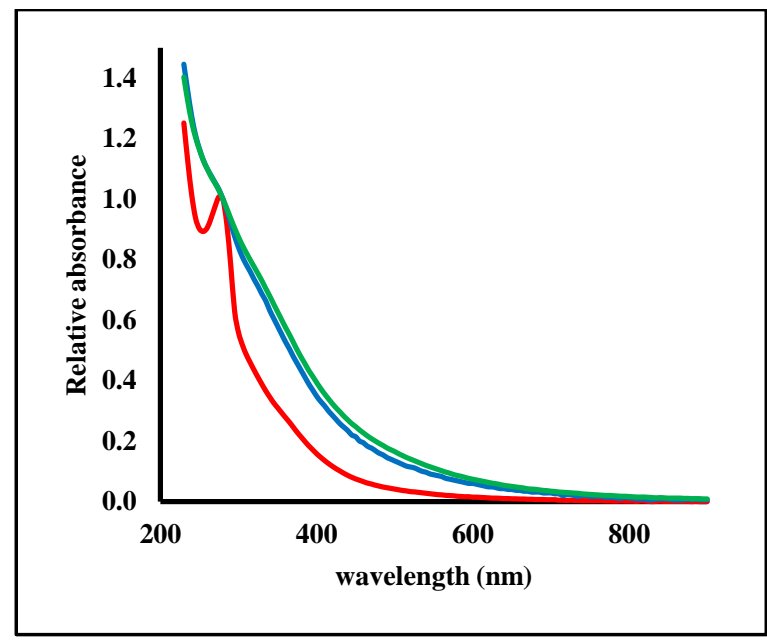

Panel A

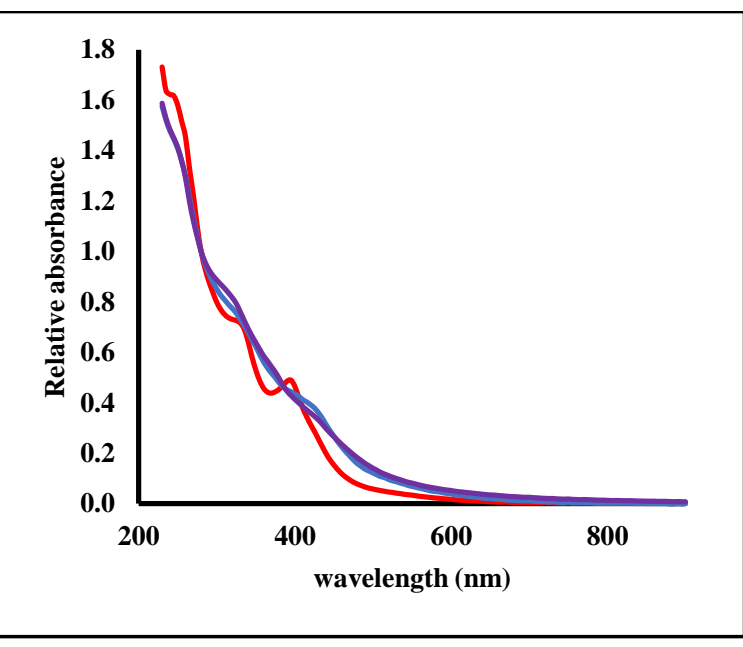

Panel B 


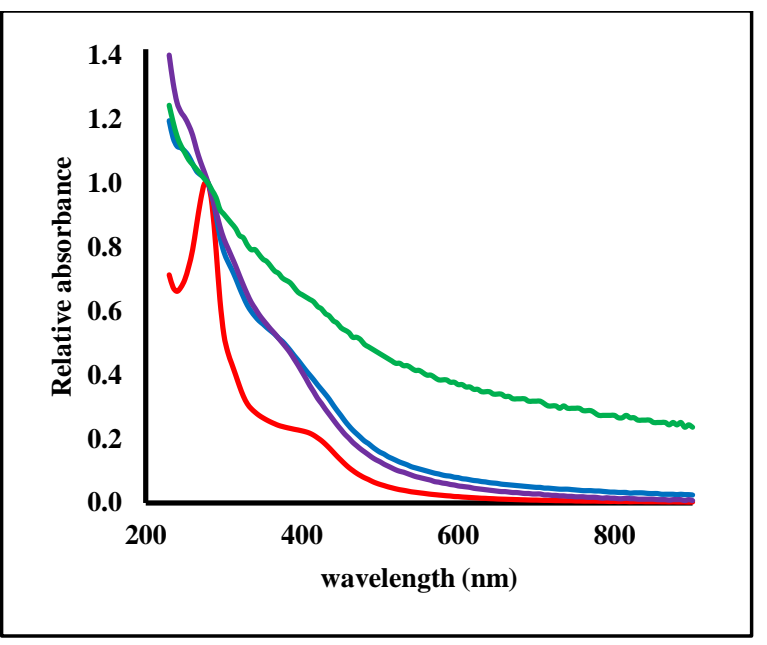

Panel C

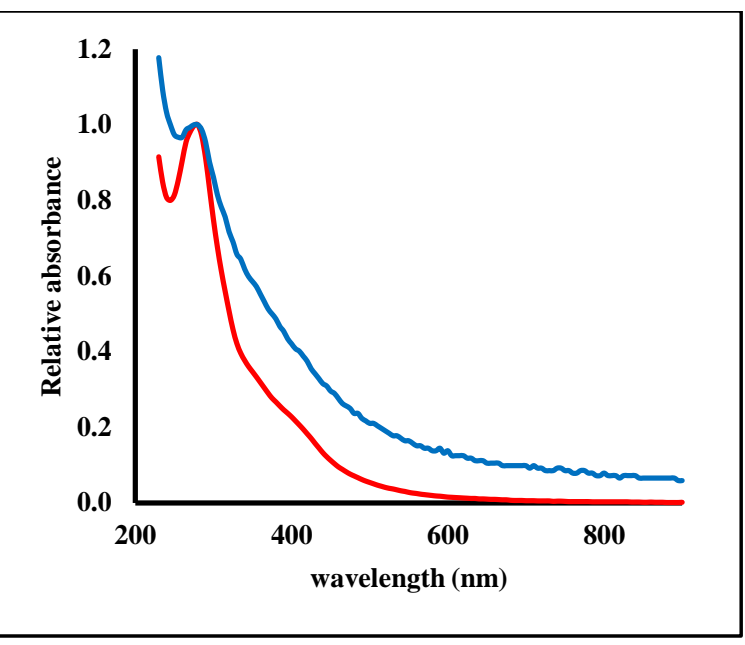

Panel D

Figure 4: UV-Vis spectra, normalized for their absorbance at $280 \mathrm{~nm}$, of purified fractions obtained from catechol (panel A), pyrogallol (panel B), L-DOPA (panel C) and dopamine (panel D). Red lines correspond to fraction I, blue lines correspond to fraction III, purple lines correspond to fraction IV and green lines correspond to fraction V.

For all four compounds involved, fraction I resulted in a light-colored, yellow to red-orange material. These fractions exhibited UV-Vis profiles with clear absorbance bands in the UV portion of the electromagnetic spectrum and with minimal absorbance in the visible portion of the electromagnetic spectrum. With the exception of the case of pyrogallol, the specific sharp absorbance peaks in the UV region of the spectra of the fractions I are similar to the absorbance bands that can be observed in the spectra of the precursor compounds. This suggests that the materials isolated in fractions I may be built from unoxidized precursor units. In addition to the sharp absorbance peak at $280 \mathrm{~nm}$, the spectra of fractions I obtained from L-DOPA and dopamine also showed an enhanced absorbance band around 400nm. For all other fractions (III, IV or V), and for the crude reaction mixtures, the portion of the spectra in the visible part of the electromagnetic spectrum was featureless showing an exponentially declining level of absorbance with increasing wavelength, as is typical for MN-like materials.

For all recorded spectra, the absorbance profiles recorded in the visible portions of the electromagnetic spectrum could be fitted according to Eq. 1 with correlation coefficients above 0.99. Table 2 presents typical results for the estimates of the decay constants k (see Eq. 1) thus obtained. In addition, Table 2 includes the ratio of the absorbance at $650 \mathrm{~nm}$ to the absorbance at $500 \mathrm{~nm}$ as suggested by Wakamatsu et al. to discriminate between eumelanin and pheomelanin. ${ }^{39}$ Figure 5 illustrates the relationship we observed between the decay constant $\mathrm{k}$ and this ratio. 


\begin{tabular}{l} 
Table 2: Overview of typical decay constants (k; see Eq. 1), of the UV-Vis spectra of the \\
purified fractions and the ratio of the absorbance at 650 to the absorbance at 500nm \\
$\left(\mathrm{A}_{650} / \mathrm{A}_{500}\right)$. \\
\hline
\end{tabular}

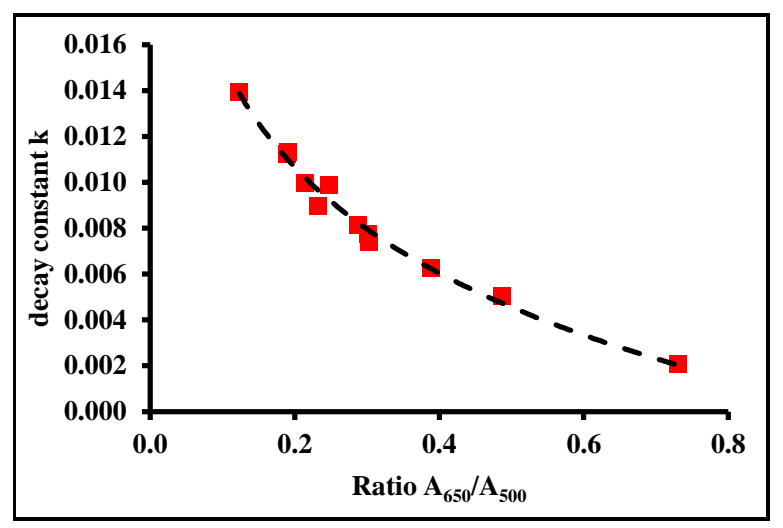

Figure 5: Relationship between the ratio of absorbance at $650 \mathrm{~nm}$ over $500 \mathrm{~nm}$ and the decay constant of the spectra of various fractions obtained from reactions involving catechol, pyrogallol, L-DOPA or dopamine.

\subsection{Fluorescence emission}

For those fractions that following their purification could be completely dissolved in water, the fluorescence emission was measured as a function of pigment concentration. The results are shown in Figure 6. 


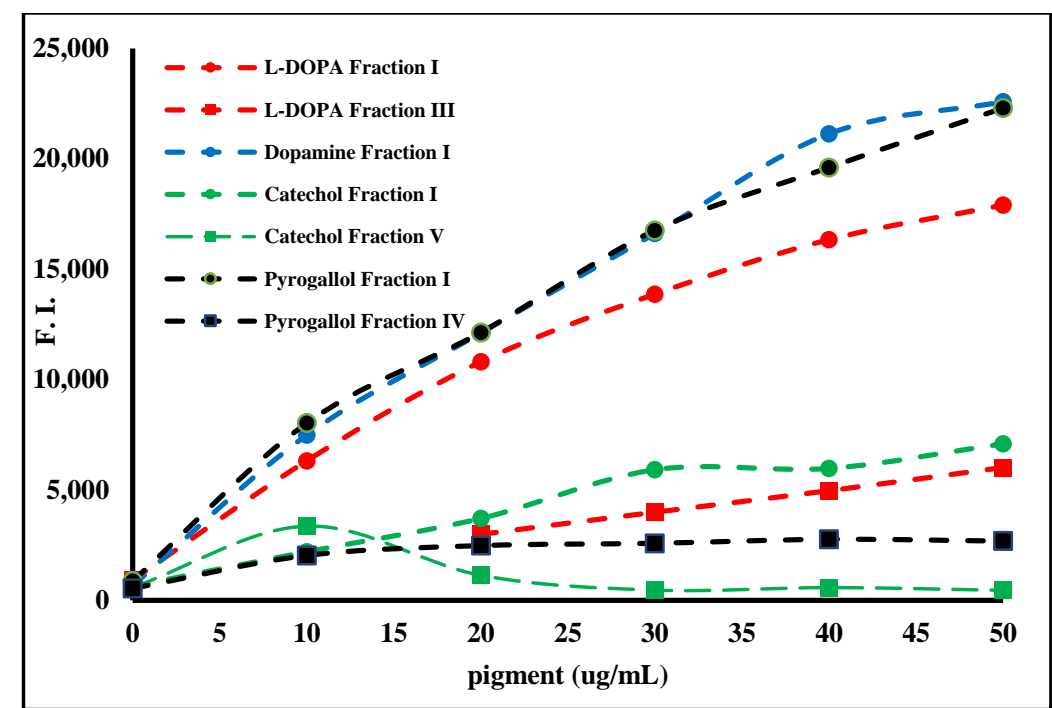

Figure 6: Concentration dependent fluorescence emission (see Materials and Methods) of various fractions obtained from catechol, pyrogallol, L-DOPA or dopamine following their dialysis and drying.

With the exception of catechol, the materials isolated in fraction I yielded a substance with concentration-dependent fluorescent properties. For the other fractions obtained, the fluorescence emission appeared to be minimal or could not be compared as these fractions did not completely dissolve in water following their dialysis and drying.

\subsection{FT-IR spectroscopic analyses}

As discussed in the experimental section, all reactions were performed and processed in duplicate. In addition, upon drying some fractions were divided into "powdery" and "sticky" sub-fractions. All these dried materials, combined with the ones from the experiments described further in this report, yielded a total of 30 FT-IR scans. Some of these spectra, grouped by precursor compound involved and normalized for their absorbance at 1,590 $\mathrm{cm}^{-1}$, are shown in Figure 7, panels A through D. 


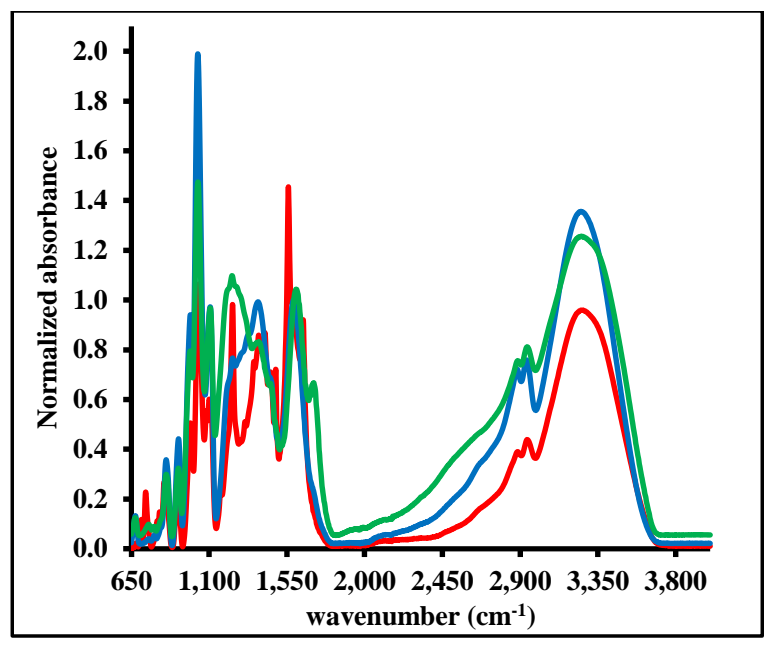

Panel A

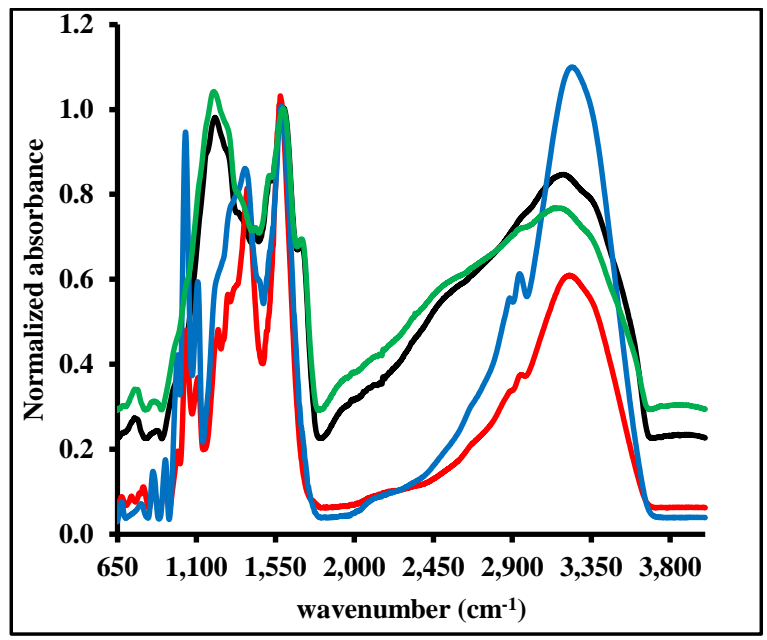

Panel C

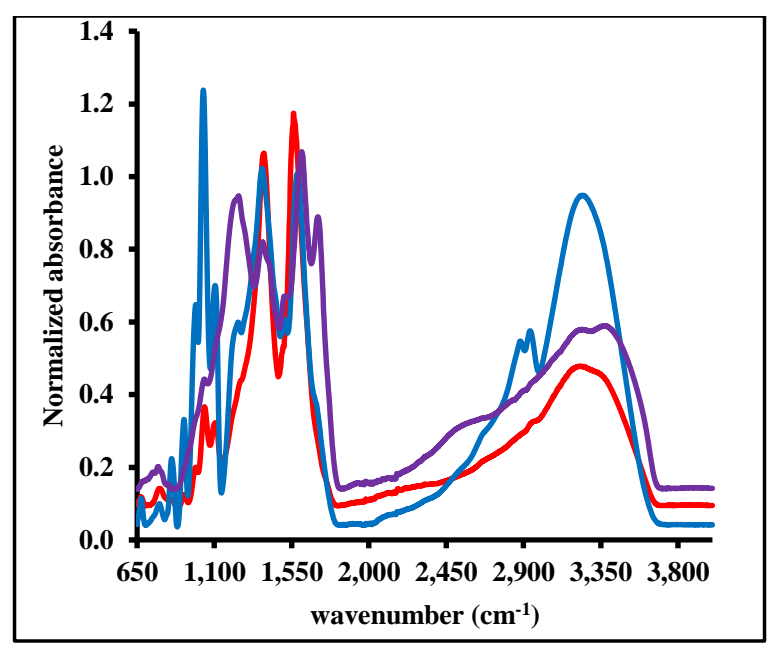

Panel B

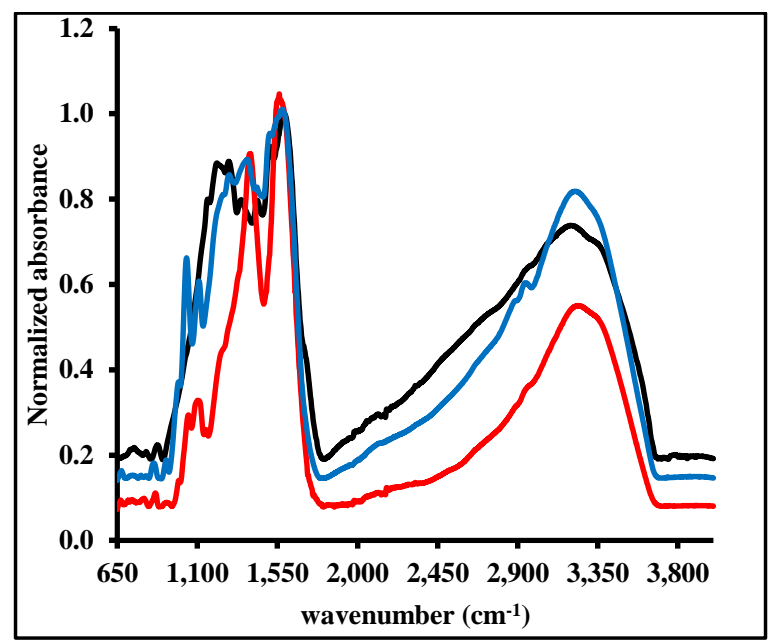

Panel D

Figure 7: FT-IR spectra, normalized for their absorbance at $1,590 \mathrm{~cm}^{-1}$, for purified fractions obtained from catechol (panel A), pyrogallol (panel B), L-DOPA (panel C) and dopamine (panel D). Red lines correspond to fraction I, blue lines correspond to fraction III, purple lines correspond to fraction IV, green lines correspond to fraction V and black lines correspond to remaining precipitate.

For the 30 FT-IR scans obtained, the absorbance values at $2,945 \mathrm{~cm}^{-1}$ were correlated with the absorbance values in the fingerprint regions $\left(650\right.$ to $\left.1,800 \mathrm{~cm}^{-1}\right)$ of these scans. Figure 8, panel A, shows one typical example of a strong correlation thus observed. Figure 8, panel B, shows a plot of the correlation coefficients $\left(\mathrm{R}^{2}\right)$ between the absorbance values at $2,945 \mathrm{~cm}^{-1}$ and the absorbance values for all the wavenumbers between 650 and $1,800 \mathrm{~cm}^{-1}$. 


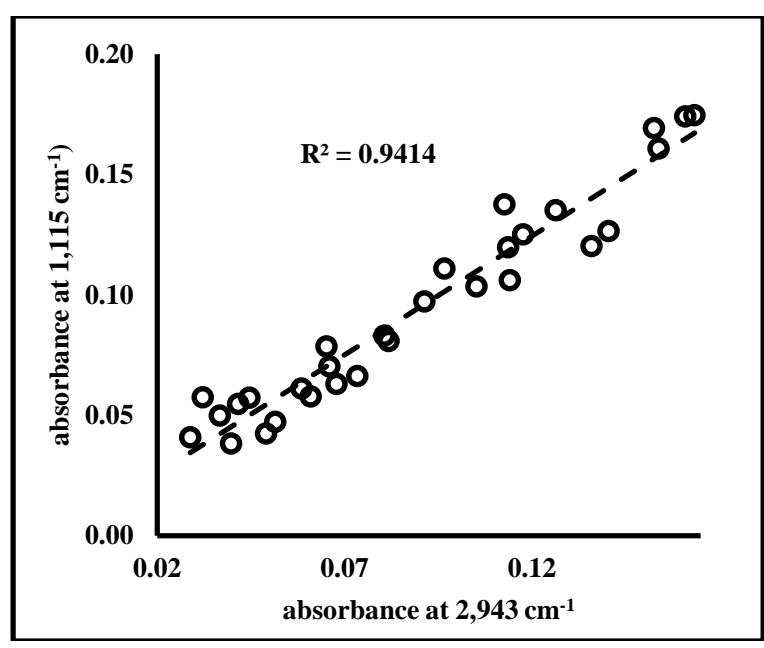

Panel A

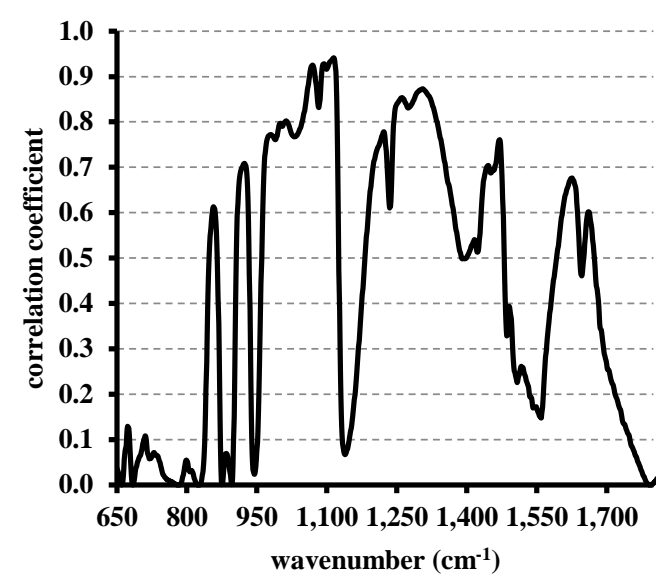

Panel B

Figure 8: For all 30 FT-IR scans obtained in the course of the experiments reported here, panel A shows the correlation between the absorbance at $2,945 \mathrm{~cm}^{-1}$ and the absorbance at $1,115 \mathrm{~cm}^{-1}$, while Panel B shows the correlation coefficients $\left(\mathrm{R}^{2}\right)$ between the absorbance values at $2,945 \mathrm{~cm}^{-1}$ and the absorbance values for the wavenumbers between 650 and $1,800 \mathrm{~cm}^{-1}$.

From Figure 8, panel B, it can be observed that strong correlation $\left(\mathrm{R}^{2}>0.90\right)$ between the absorbance at 2,945 $\mathrm{cm}^{-1}$ and the absorbance values for wavenumbers between 1,065 and 1,120 $\mathrm{cm}^{-1}$ and between 1,250 and $1,300 \mathrm{~cm}^{-1}$ exist.

\subsection{Evaluation of the fractionation process: L-DOPA and dopamine}

As outlined in Materials and Methods, the washing process following the co-precipitation of $\mathrm{MN}$-like materials onto $\mathrm{CaCO}_{3}$ was further evaluated using L-DOPA and dopamine. Two different processes were compared: A) repeated washes with water and B) washes with water interspersed with washes involving 0.1 $\mathrm{N} \mathrm{HCl}$. Figure 9, panels A and B, present the "darkness" of the supernatants, evaluated through the calculation of the AUC of the visible spectrum between 400 and $900 \mathrm{~nm}$ as outlined in Materials and Methods, at every stage of the washing process. 


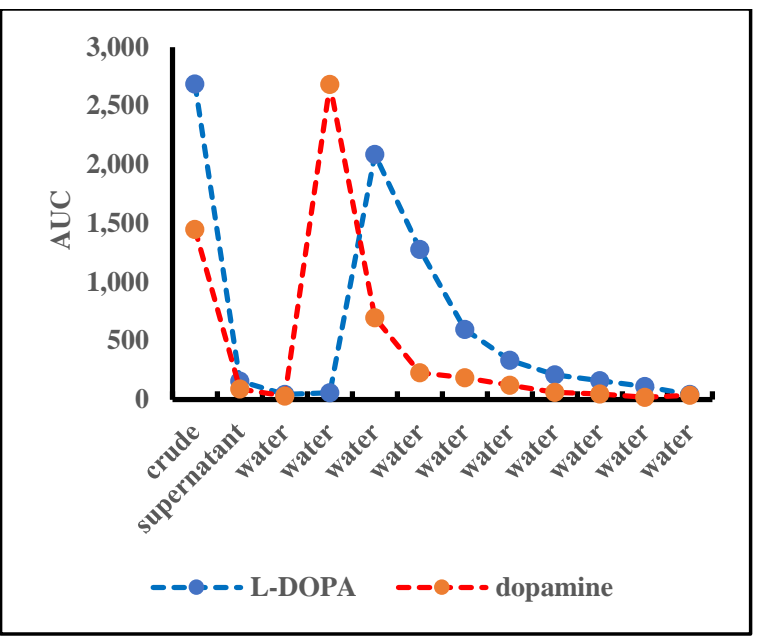

Panel A

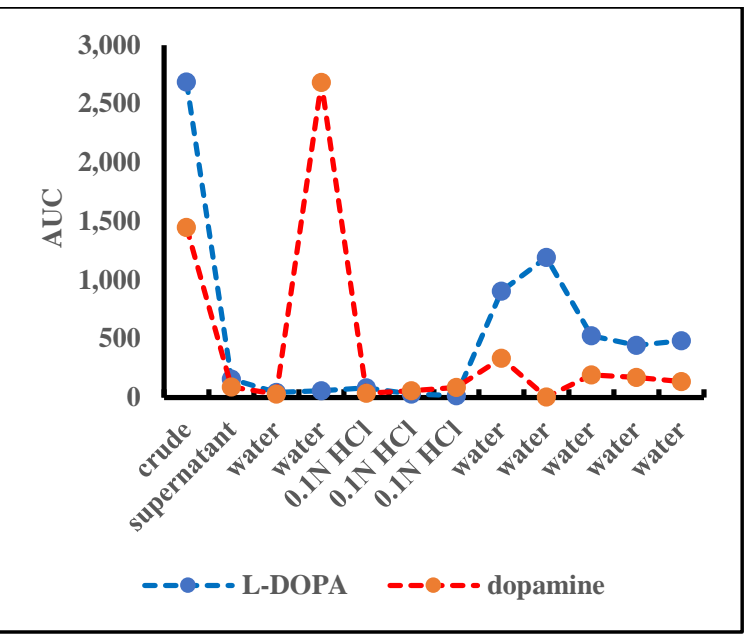

Panel B

Figure 9: "Darkness" (as estimated from AUC values as discussed in Materials and Methods) of the crude reaction mixtures involving L-DOPA or dopamine and their supernatants following co-precipitation on $\mathrm{CaCO} 3$ and subsequent washes with water (panel A) or subsequent washes with water interspersed with washes with $0,1 \mathrm{~N} \mathrm{HCl}$ (panel B).

The observations made in these experiments are in accordance with what was observed for the previous experiments: a) at the end of the reaction, the crude reaction mixtures are very dark in appearance, b) most of the dark materials were co-precipitated onto $\mathrm{CaCO}_{3}$, c) the initial washes with water (once for dopamine or twice for L-DOPA) yielded near colorless supernatants and d) a subsequent wash with water yielded a supernatant with a very dark appearance. Continued washing of the precipitate with water (Figure 9, panel A) yielded supernatants with decreasing darkness in appearance. In the case of L-DOPA little to no precipitate remained after all these washes. In the case of dopamine, some black-colored precipitate remained after all these washes and this precipitate could be lyophilized and a FT-IR scan was obtained.

The interspersed washes with $0.1 \mathrm{~N} \mathrm{HCl}$ (Figure 9, panel B) yielded near colorless supernatants, but subsequent washes with water yielded some (L-DOPA) or minimal (dopamine) amounts of dark-colored substances. In both cases some amounts of black-colored precipitate remained and these could be lyophilized and FT-IR scans were obtained. For both compounds, some part of the crude reaction mixture was dialyzed and lyophilized and FT-IR scans were recorded. Figure 10, panels A and B, show some of the FT-IR scans, normalized for their absorbance at $1,590 \mathrm{~cm}^{-1}$, thus obtained. 


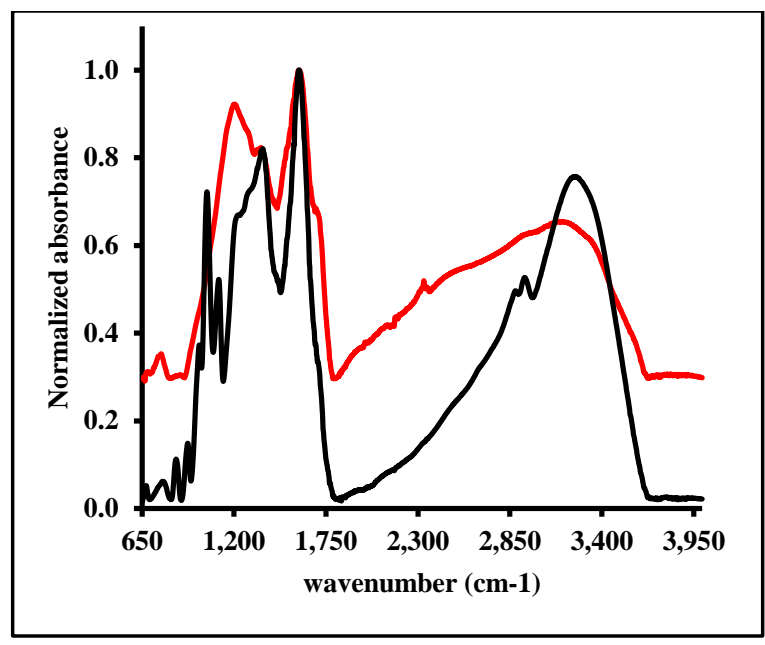

Panel A

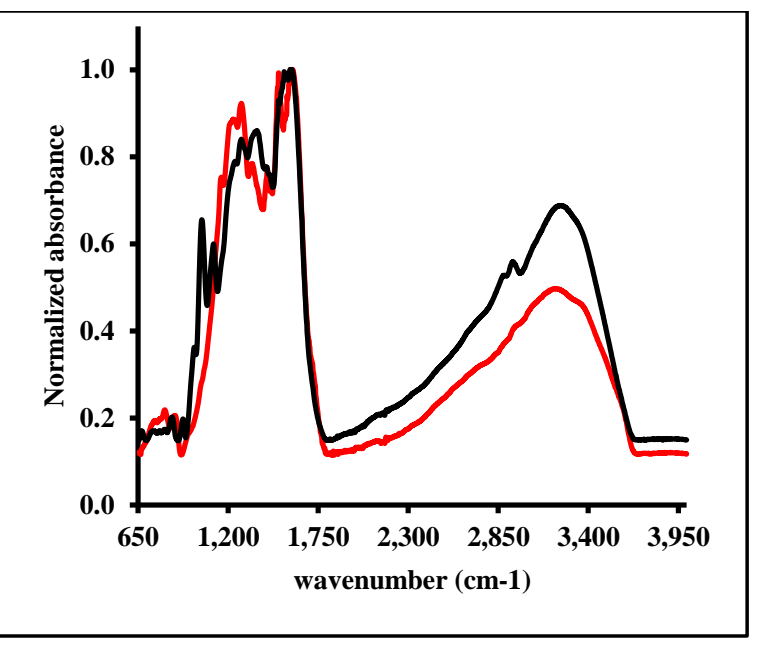

Panel B

Figure 10: FT-IR scans of dialyzed crude reaction mixtures (black lines) and water/acid-washed precipitates (red lines) of L-DOPA-based materials (panel A) or dopamine-based materials (panel B) as discussed in Materials and Methods.

The FT-IR spectra of the precipitates obtained from dopamine following washing with water or washing with water interspersed with $0.1 \mathrm{~N} \mathrm{HCl}$ were qualitatively similar and only the scan obtained for the water/acid-washed precipitate is shown in Figure 10, panel B. For both compounds, the main qualitative difference between the FT-IR scans of the dialyzed crude mixture and the washed precipitates are the increased relative absorbance at the twin peaks around 2,885 and 2,945 $\mathrm{cm}^{-1}$ and the relative increased absorbance around $1,100 \mathrm{~cm}^{-1}$. In addition, the FT-IR scan of the water/acid washed precipitated obtained from L-DOPA has an enhanced absorbance shoulder around $1,700 \mathrm{~cm}^{-1}$.

\section{Discussion}

The current observations are in accordance with our earlier observations. ${ }^{2}$ The typical conditions to synthesize MNs, air-oxidation in an alkaline environment, leads to the generation of a light-colored substance in addition to the typical dark substances. This lighter-colored substance appears to be associated with the darker-colored materials through non-covalent interactions and, judging from the experiments associated with Figure 10, can not be removed through dialysis. The FT-IR spectra of the dialyzed crude reaction mixtures shown in Figure 10 possess features that overlap with features observed in the FT-IR spectra of the purified fractions I (see Figure 7, panels C or D). The novel co-precipitation work-up procedure (see Scheme 1) we 
employed allows for a simple fractionation of the lighter-colored substance from the darkercolored materials. Overall, the current observations strengthen our hypothesis that $\mathrm{MN}$ materials are built from at least two types of substances: 1) a soluble, yellow-to-orange colored component, rich in unoxidized precursors and 2) an insoluble, dark component. In addition, our earlier experiments ${ }^{2-3}$ and other reports ${ }^{15}, 19-20,32,40-42$ indicate that the ratio of the various reaction products generated, and/or their physic-chemical properties, may depend on the reaction conditions applied.

The observations discussed in this report confirm and expand, by the inclusion of catechol and pyrogallol, our earlier reports on the possibility to precipitate $\mathrm{MN}$-like materials on mineral substrates like $\mathrm{CaCO}_{3}$ post synthesis. ${ }^{2}$ Similar co-precipitations can be achieved on other minerals like $\mathrm{Ca}_{3}\left(\mathrm{PO}_{4}\right)_{2}, \mathrm{Ca}(\mathrm{OH})_{2}$ or the corresponding magnesium salts. Biocompatible materials like PDA have been studied for their potential effect on the polymorphism and physical dimensions of $\mathrm{CaCO}_{3}$ materials. ${ }^{43}$ The observations made during the work-up of the larger reaction mixtures and during the experiments associated with Figures 9 and 10 indicate that the co-precipitation process and subsequent washing of the MN-like materials allows for a fractionation of the MN precipitate, akin to the "peeling off" of layers of materials. The work-up process outlined in Scheme 1 may have fractioned MN-like materials based upon differences in adhesiveness. Fraction I constituting the least adhesive portion, Fraction III constituting an intermediate-adhesive portion and Fraction IV (pyrogallol), Fraction V (catechol) or the remaining precipitate (L-DOPA or dopamine) constituting the strongest adhesive portion of the materials generated. This "peeling off" of layers of precipitated material may be similar to the fractionation of MN-like materials into nanoparticle subunits through the washing with $\mathrm{NaOH}$ solutions as described elsewhere. ${ }^{20,33}$ The observations we made does indicate that, apart from the details of the synthesis conditions, the details of the work-up of the reaction products should be carefully controlled.

Our observations regarding the differences to what extent MN-like materials derived from LDOPA or dopamine adhere to $\mathrm{CaCO}_{3}$ compared to $\mathrm{MN}$-like materials derived from catechol or pyrogallol (see Figure 2) are in line with other reports on the difference in adhesiveness of amine-containing $\mathrm{MN}$-like materials compared to nitrogen-free $\mathrm{MN}$-like materials. ${ }^{44} \mathrm{With}$ the notable exception of pyrogallol, the washing of the precipitate with $0.1 \mathrm{~N} \mathrm{HCl}$ (fraction IV; meant

\footnotetext{
${ }^{*}$ As observed during other experiments in our laboratory.
} 
to dissolve the precipitated $\mathrm{CaCO}_{3}$ ) did not induce any noticeable resuspension of precipitated material. Subsequent washes with water will release material from the precipitate but, as shown in Figure 9, not as readily as when the precipitate does not undergo a wash with $0.1 \mathrm{~N} \mathrm{HCl}$. Taken together, these observations suggest that washing MN materials with an acidic solution may enhance their adhesiveness.

For all four precursors used, the initial washing of the precipitate (see Scheme 1) with water did not follow a predictable pattern. A first wash with water (Fraction II) did not release any major amounts of material, but the second wash of the precipitate (Fraction III) resulted in the resuspension of significant amounts of dark material. After the first wash one can expect that the bulk of the remaining buffer salts and the excess $\mathrm{CaCl}_{2}$ have been washed away. The resuspension of some of the dark material during the second wash with water could be due to the changes in $\mathrm{pH}$ and/or ionic strength that occurred after the first wash with water. Similarly, the dialysis process, with its repeated changes of water, will lead to changes in $\mathrm{pH}$ and/or ionic strength of the environment of the material to be purified. Our observations regarding the physical instability, i.e., the aggregation and precipitation, of some fractions during the dialysis process could be induced by these changes in $\mathrm{pH}$ and/or ionic strength during the dialysis process. In this context it is worth noting the experiments discussed by Hong et al. ${ }^{35}$ In their studies they coated solid substrates with dopamine-based materials and observed that: a) the coating could be disassembled through the washing with $\mathrm{NaOH}$ solutions and $\mathrm{b}$ ) that a reassembly could be achieved by washing with $\mathrm{K}^{+}$ions. The integrity of the $\mathrm{MN}$ assembly may be affected by external conditions like $\mathrm{pH}$, cations, etc. and in part be determined through cation- $\pi$ interactions as discussed by Hong et al.

For all precursors involved, Fraction I appeared as a dark-orange (L-DOPA and dopamine) or dark-brown (catechol and pyrogallol) solution. As shown in Figure 3, the materials are yellow in color, exhibiting dark-orange or dark-brown appearances when dissolved at a higher concentration. This yellow color may be due to the absorbance peaks or bands close to $400 \mathrm{~nm}$ that can be seen in the UV-Vis spectra of these fractions (see Figure 4). In the case of L-DOPAbased materials, one could attribute the presence of this lighter-colored substance to an enrichment of DHICA units in the material. ${ }^{45}$ But such a discussion does not hold for the lightercolored fractions obtained from the other three precursors. The lighter colors are also reflected in the higher values of the decay constants (k see Eq.1) and the lower ratio of the absorbance at 
$650 \mathrm{~nm}$ over the absorbance at 500nm (see Table 2) of the various Fraction I materials. Some of these absorbance ratio values are within the range suggested by Wakamatsu et al. to differentiate pheomelanin from eumelanin. ${ }^{39}$ The value of the decay constant $\mathrm{k}$ (see Eq.1) serves as an alternative parameter to describe the "color" of the material, with lighter-colored materials exhibiting higher values of $\mathrm{k}$. Both parameters have the advantage of being independent of the sample concentration. For some fractions the values of both parameters are listed in Table 2 and Figure 5 illustrates that both parameters are related to each other. The results presented in Table 2 indicate that all fractions obtained from pyrogallol appear to be relatively light in color. No fraction obtained from pyrogallol exhibited a black appearance as determined by visual observations.

For the materials obtained in fractions III, IV or V, the absorbance profiles in the visible range of the electromagnetic spectrum exhibited exponentially-declining absorbance values with increasing wavelength (see Figure 4). Such exponentially declining absorbance profiles, as typically observed for MN-like materials, are attributed to the presence of an array of different chemical entities, each with their own absorbance profiles. The superimposition of these various individual absorbance profile then generate the overall absorbance profile of the materials; the so-called chemical disorder model. ${ }^{27}$ Despite the heterogeneity in the chemistry of the precursors used, all fractions of the $\mathrm{MN}$-like materials generated yielded exponentially declining absorbance profiles. In addition, countless experiments from our (or others') laboratory, reported and unreported, involving the same precursors, but applying different reaction conditions (different concentrations of precursors; different concentrations of base; addition of other molecules; etc.), yielded materials with similar types of absorbance profiles. If "chemical disorder" is the underlying cause of these profiles, then this would constitute a remarkable coincidence that under all these varying reaction conditions, the various chemical entities would readily be generated in the necessary ratios that would lead to the typical exponentially declining absorbance profiles. A different explanation for the absorbance profiles/dark colors of MN-like materials has been suggested and discussed elsewhere..$^{29-30}$

With the exception of catechol, the materials present in any fraction I exhibited fluorescent emission properties (see Figure 6) and these observations are in line with the fluorescent emission properties described for eumelanin or PDA-based materials ${ }^{14,20-25}$, Sepia melanin ${ }^{33}$ and 
pheomelanin $^{46}$. However, our work indicates that the fluorescence of MN-like materials may be associated with a subfraction of the material; not the MN material in its entirety.

The FT-IR spectra shown in Figures 7 and 10 are in agreement with the multitude of reports that have been published in the past. ${ }^{47-49}$ and references within All spectra exhibit a very broad absorbance band maxed between 3,150 and $3,350 \mathrm{~cm}^{-1}$, broad bands or distinct peaks between 1,500 and $1,600 \mathrm{~cm}^{-1}$, between 1,425 and $1,485 \mathrm{~cm}^{-1}$, between 1,200 and $1,400 \mathrm{~cm}^{-1}$, between 990 and $1,150 \mathrm{~cm}^{-1}$ and a cluster of smaller peaks between 650 and $950 \mathrm{~cm}^{-1}$. Some unique features observed are: a) sharp peaks in the spectrum of fraction I obtained from catechol, b) a peak or shoulder around $1,700 \mathrm{~cm}^{-1}$ in the spectra of fraction IV obtained from pyrogallol, fraction $\mathrm{V}$ obtained from catechol and fraction $\mathrm{V}$ and the remaining precipitate obtained from LDOPA. The latter absorbance feature is typically attributed to carbonyl functionalities of carboxylic acids or ketones/aldehydes. ${ }^{50}$ In the cases of catechol and pyrogallol, the carbonyl could be from ketone functionalities and may indicate the presence of quinone functionalities. In the case of L-DOPA, the peak or shoulder at $1,700 \mathrm{~cm}^{-1}$ may indicate the presence of the carboxylic acid in the materials present in fraction $\mathrm{V}$ or the remaining precipitate. These two fractions were obtained following the washing with $0.1 \mathrm{~N} \mathrm{HCl}$. We assume that the absence of a peak or shoulder at $1,700 \mathrm{~cm}^{-1}$ in fractions I and III obtained from L-DOPA may indicate that these fractions contain the carboxylic acid in its carboxylate form as the $\mathrm{Na}^{+}$and/or $\mathrm{Ca}^{2+} \mathrm{Salt}$. A more challenging interpretation of the FT-IR data we collected is posed by the two smaller peaks at wavenumbers of about 2,885 and $2,945 \mathrm{~cm}^{-1}$ which can be observed in some, but not all the spectra we recorded; independent of the precursor involved (see Figure 7). These two peaks have

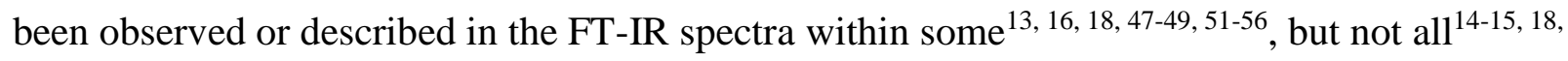
27, 40, 47-48, 56-60 other reports. The absorbance at these two twin wavenumbers are typically attributed to aliphatic C-H stretching. This could be expected for L-DOPA- or dopamine-based materials, but not for catechol- or pyrogallol-based materials. However, clear signals at these two wavenumbers were observed in only a few spectra of L-DOPA- or dopamine-based materials, in all the spectra of the catechol-based materials and in the spectrum of fraction III of the pyrogallol-based materials. It is of interest to note that: a) the presence of absorbance bands at wavenumbers of about 2,885 and 2,945 $\mathrm{cm}^{-1}$ were recognized, and even described as distinctive for neuromelanin ${ }^{13,61}$ and $b$ ) infrared spectra obtained from natural or synthetic pheomelanin materials exhibited clear signals at about 2,855 and 2,930 $\mathrm{cm}^{-1}$ and strong absorbance bands 
around $1,100 \mathrm{~cm}^{-1} \cdot{ }^{48,54}$ In this latter case, these absorbance features had been attributed to the thiazole or benzothiazole moieties of pheomelanin ${ }^{54}$, but this obviously can not be an explanation for our observations. The absorbance at $2,945 \mathrm{~cm}^{-1}$ correlates strongly with the absorbances between 1,065 and 1,120 $\mathrm{cm}^{-1}$ and between 1,250 and 1,300 $\mathrm{cm}^{-1}$ (see Figure 8). These wavenumber ranges do correspond to the absorbance range one may expect for $\mathrm{C}-\mathrm{O}$ stretching from alcohol or phenol groups. ${ }^{50}$ The significance of our observations regarding these features in the FT-IR spectra is currently unknown.

For one reaction involving L-DOPA, fraction IV was dialyzed and lyophilized. But this material would dry into an "oily" substance and only a crude UV-Vis spectrum could be obtained (included in Figure 4, panel C). Similarly, some other fractions yielded "sticky" or "oily" substances. At this point it is unclear whether the physical aspects of these "sticky" MNlike materials are related to the "liquid-like" MN reported elsewhere ${ }^{41}$ or are similar to the physical properties of the oxidation products of 5-S-cysteinyldopa which have been described as yellow, orange, orange-brown or brown oils. ${ }^{62}$

In conclusion, our results indicate that the air-oxidation in alkaline environment of catecholic precursors results in the production of MN-like materials in which is embedded at least one substance with physic-chemical properties unlike typical MN-materials: soluble in water, relatively light in color, fluorescent and non-adhesive. The co-precipitation work-up process we developed allows for an easy partial purification of this substance. In addition, the work-up process with its repeated washes appears to allow for a fractionation ("peeling off") of precipitated $\mathrm{MN}$ based upon differences in adhesive properties of its different layers. A careful fractionation of the MN material generated is crucial for the development and optimization of any application of MN-like materials; particularly if one wants to relate functionality to chemical structure. Many of the properties (color, fluorescence or FT-IR absorbance features) of some of the fractions we obtained are very similar to what has been associated with pheomelanin and to a lesser extent with neuromelanin. Whether our observations have any relevance to the physiology or biochemistry of MNs in vivo remains to be seen. Our observations do suggest that when studying the biochemistry or physiology of MNs one should look beyond the dark colors typically displayed by MN-like materials. 


\section{References}

1.Vercruysse, K., Evaluating the "Darkness" of Melanin Materials, Personal Communication,

10.26434/chemrxiv.12762179.v1, 2020

2.Vercruysse, K.; Govan, V., Melanogenesis: A Search for Pheomelanin and Also, What Is Lurking Behind Those Dark Colors?, Personal Communication, 10.26434/chemrxiv.11418075.v1, 2019

3.Vercruysse, K.; Govan, V.; Fortner, S., Kinetic Study of the Melanogenesis from Select Catecholamines in the Presence of L-Cysteine or Other Amino Acids, Personal Communication, 10.26434/chemrxiv.12678860.v1, 2020 4.Solano, F., Melanins: Skin Pigments and Much More-Types, Structural Models, Biological Functions, and Formation Routes. New Journal of Science 2014, 2014, 498276.

5.Maranduca, M. A.; Branisteanu, D.; Serban, D. N.; Branisteanu, D. C.; Stoleriu, G.; Manolache, N.; Serban, I. L., Synthesis and physiological implications of melanic pigments (Review). Oncol Lett 2019, 17 (5), 4183-4187. 6.Eisenman, H. C.; Greer, E. M.; McGrail, C. W., The role of melanins in melanotic fungi for pathogenesis and environmental survival. Appl Microbiol Biotechnol 2020, 104 (10), 4247-4257.

7.McNamara, M. E.; Rossi, V.; Slater, T. S.; Rogers, C. S.; Ducrest, A. L.; Dubey, S.; Roulin, A., Decoding the Evolution of Melanin in Vertebrates. Trends in Ecology \& Evolution 2021, 36 (5), 430-443.

8.d'Ischia, M.; Wakamatsu, K.; Napolitano, A.; Briganti, S.; Garcia-Borron, J. C.; Kovacs, D.; Meredith, P.; Pezzella, A.; Picardo, M.; Sarna, T.; Simon, J. D.; Ito, S., Melanins and melanogenesis: methods, standards, protocols. Pigment Cell Melanoma Res 2013, 26 (5), 616-33.

9.Cao, W.; Zhou, X.; McCallum, N. C.; Hu, Z.; Ni, Q. Z.; Kapoor, U.; Heil, C. M.; Cay, K. S.; Zand, T.; Mantanona, A. J.; Jayaraman, A.; Dhinojwala, A.; Deheyn, D. D.; Shawkey, M. D.; Burkart, M. D.; Rinehart, J. D.; Gianneschi, N. C., Unraveling the Structure and Function of Melanin through Synthesis. J Am Chem Soc 2021, 143 (7), 26222637.

10.Simon, J. D.; Peles, D. N., The red and the black. Acc Chem Res 2010, 43 (11), 1452-60.

11.Ni, Q. Z.; Sierra, B. N.; La Clair, J. J.; Burkart, M. D., Chemoenzymatic elaboration of the Raper-Mason pathway unravels the structural diversity within eumelanin pigments. Chem Sci 2020, 11 (30), 7836-7841. 12.Micillo, R.; Panzella, L.; Iacomino, M.; Prampolini, G.; Cacelli, I.; Ferretti, A.; Crescenzi, O.; Koike, K.; Napolitano, A.; d'Ischia, M., Eumelanin broadband absorption develops from aggregation-modulated chromophore interactions under structural and redox control. Sci Rep 2017, 7, 41532.

13.Bridelli, M. G.; Tampellini, D.; Zecca, L., The structure of neuromelanin and its iron binding site studied by infrared spectroscopy. FEBS Lett 1999, 457 (1), 18-22.

14.Zheng, W.; Fan, H.; Wang, L.; Jin, Z., Oxidative Self-Polymerization of Dopamine in an Acidic Environment. Langmuir 2015, 31 (42), 11671-11677.

15.Bronze-Uhle, E. S.; Paulin, J. V.; Piacenti-Silva, M.; Battocchio, C.; Rocco, M. L. M.; Graeff, C. F. d. O., Melanin synthesis under oxygen pressure. Polymer International 2016, 65 (11), 1339-1346.

16.El-Naggar, N. E.; El-Ewasy, S. M., Bioproduction, characterization, anticancer and antioxidant activities of extracellular melanin pigment produced by newly isolated microbial cell factories Streptomyces glaucescens NEAEH. Sci Rep 2017, 7, 42129. 
17.Binns, F.; King, J. A.; Mishra, S. N.; Percival, A.; Robson, N. C.; Swan, G. A.; Waggott, A., Studies related to the chemistry of melanins. 13. Studies on the structure of dopamine-melanin. Journal of the Chemical Society. Perkin transactions 1 1970, 15, 2063-70.

18.Liebscher, J.; Mrowczynski, R.; Scheidt, H. A.; Filip, C.; Hadade, N. D.; Turcu, R.; Bende, A.; Beck, S., Structure of polydopamine: a never-ending story? Langmuir 2013, 29 (33), 10539-48.

19.Liebscher, J., Chemistry of Polydopamine - Scope, Variation, and Limitation. European Journal of Organic Chemistry 2019, 2019 (31-32), 4976-4994.

20.Ju, K. Y.; Fischer, M. C.; Warren, W. S., Understanding the Role of Aggregation in the Broad Absorption Bands of Eumelanin. ACS nano 2018, 12 (12), 12050-12061.

21.Ball, V., Polydopamine Nanomaterials: Recent Advances in Synthesis Methods and Applications. Front Bioeng Biotechnol 2018, 6, 109.

22.Yildirim, A.; Bayindir, M., Turn-on Fluorescent Dopamine Sensing Based on in Situ Formation of Visible Light Emitting Polydopamine Nanoparticles. Analytical Chemistry 2014, 86 (11), 5508-5512.

23.Zhang, X.; Wang, S.; Xu, L.; Feng, L.; Ji, Y.; Tao, L.; Li, S.; Wei, Y., Biocompatible polydopamine fluorescent organic nanoparticles: facile preparation and cell imaging. Nanoscale 2012, 4 (18), 5581-4.

24.Meredith, P.; Powell, B. J.; Riesz, J.; Nighswander-Rempel, S. P.; Pederson, M. R.; Moore, E. G., Towards structure-property-function relationships for eumelanin. Soft Matter 2006, 2 (1), 37-44.

25.Chen, T. P.; Liu, T.; Su, T. L.; Liang, J., Self-Polymerization of Dopamine in Acidic Environments without Oxygen. Langmuir 2017, 33 (23), 5863-5871.

26.Meredith, P.; Sarna, T., The physical and chemical properties of eumelanin. Pigment Cell Res 2006, 19 (6), 57294.

27.Tran, M. L.; Powell, B. J.; Meredith, P., Chemical and structural disorder in eumelanins: a possible explanation for broadband absorbance. Biophys J 2006, 90 (3), 743-52.

28.Mostert, A. B., Melanin, the What, the Why and the How: An Introductory Review for Materials Scientists Interested in Flexible and Versatile Polymers. Polymers (Basel) 2021, 13 (10).

29.Chen, C.-T.; Chuang, C.; Cao, J.; Ball, V.; Ruch, D.; Buehler, M. J., Excitonic effects from geometric order and disorder explain broadband optical absorption in eumelanin. Nature Communications 2014, 5 (1), 3859.

30.Stark, K. B.; Gallas, J. M.; Zajac, G. W.; Golab, J. T.; Gidanian, S.; McIntire, T.; Farmer, P. J., Effect of stacking and redox state on optical absorption spectra of melanins -- comparison of theoretical and experimental results. $J$ Phys Chem B 2005, 109 (5), 1970-7.

31.Bungeler, A.; Hamisch, B.; Huber, K.; Bremser, W.; Strube, O. I., Insight into the Final Step of the Supramolecular Buildup of Eumelanin. Langmuir 2017, 33 (27), 6895-6901.

32.Bungeler, A.; Hamisch, B.; Strube, O. I., The Supramolecular Buildup of Eumelanin: Structures, Mechanisms, Controllability. Int J Mol Sci 2017, 18 (9).

33.Ju, K. Y.; Kang, J.; Chang, J. H.; Lee, J. K., Clue to Understanding the Janus Behavior of Eumelanin: Investigating the Relationship between Hierarchical Assembly Structure of Eumelanin and Its Photophysical Properties. Biomacromolecules 2016, 17 (9), 2860-72. 
34.Klosterman, L.; Bettinger, C. J., Calcium-Mediated Control of Polydopamine Film Oxidation and Iron Chelation. Int J Mol Sci 2016, 18 (1).

35.Hong, S.; Wang, Y.; Park, S. Y.; Lee, H., Progressive fuzzy cation- $\pi$ assembly of biological catecholamines. Science Advances 2018, 4 (9), eaat7457.

36.Alfieri, M. L.; Panzella, L.; Oscurato, S. L.; Salvatore, M.; Avolio, R.; Errico, M. E.; Maddalena, P.; Napolitano, A.; D'Ischia, M., The Chemistry of Polydopamine Film Formation: The Amine-Quinone Interplay. Biomimetics (Basel) 2018, 3 (3).

37.d'Ischia, M., Melanin-Based Functional Materials. Int J Mol Sci 2018, 19 (1).

38.Jin, Z.; Fan, H., The modulation of melanin-like materials: methods, characterization and applications. Polymer International 2016, 65 (11), 1258-1266.

39.Wakamatsu, K.; Nagao, A.; Watanabe, M.; Nakao, K.; Ito, S., Pheomelanogenesis is promoted at a weakly acidic pH. Pigment Cell Melanoma Res 2017, 30 (3), 372-377.

40.Ju, K.-Y.; Lee, Y.; Lee, S.; Park, S. B.; Lee, J.-K., Bioinspired Polymerization of Dopamine to Generate Melanin-Like Nanoparticles Having an Excellent Free-Radical-Scavenging Property. Biomacromolecules 2011, 12 (3), 625-632.

41.Chen, Y.-c.; Kendall, T.; Yip, P.; Davy, A.; Sefcik, J.; Sutter, J. U., Influence of Ions and pH on the Formation of Solid- and Liquid-like Melanin. ACS Omega 2020, 5 (39), 25059-25068.

42.Della Vecchia, N. F.; Avolio, R.; Alfè, M.; Errico, M. E.; Napolitano, A.; d'Ischia, M., Building-Block Diversity in Polydopamine Underpins a Multifunctional Eumelanin-Type Platform Tunable Through a Quinone Control Point. Advanced Functional Materials 2013, 23 (10), 1331-1340.

43.Vidallon, M. L. P.; Yu, F.; Teo, B. M., Controlling the Size and Polymorphism of Calcium Carbonate Hybrid Particles Using Natural Biopolymers. Crystal Growth \& Design 2020, 20 (2), 645-652.

44.Lim, C.; Huang, J.; Kim, S.; Lee, H.; Zeng, H.; Hwang, D. S., Nanomechanics of Poly(catecholamine) Coatings in Aqueous Solutions. Angew Chem Int Ed Engl 2016, 55 (10), 3342-6.

45.Micillo, R.; Panzella, L.; Koike, K.; Monfrecola, G.; Napolitano, A.; d'Ischia, M., "Fifty Shade“ of Black and Red or How Carboxyl Groups Fine Tune Eumelanin and Pheomelanin Properties. International Journal of Molecular Sciences 2016, 17.

46.Nighswander-Rempel, S. P., Quantitative fluorescence spectra and quantum yield map of synthetic pheomelanin. Biopolymers 2006, 82 (6), 631-637.

47.Pralea, I. E.; Moldovan, R. C.; Petrache, A. M.; Ilies, M.; Heghes, S. C.; Ielciu, I.; Nicoara, R.; Moldovan, M.; Ene, M.; Radu, M.; Uifalean, A.; Iuga, C. A., From Extraction to Advanced Analytical Methods: The Challenges of Melanin Analysis. Int J Mol Sci 2019, 20 (16).

48.Liu, Y.; Hong, L.; Wakamatsu, K.; Ito, S.; Adhyaru, B.; Cheng, C. Y.; Bowers, C. R.; Simon, J. D., Comparison of structural and chemical properties of black and red human hair melanosomes. Photochem Photobiol 2005, 81 (1), 135-44.

49.Mbonyiryivuze, A.; Mwakikunga, B.; Mokhotjwa Dhlamini, S.; Maaza, M., Fourier Transform Infrared Spectroscopy for Sepia Melanin. Physics and Materials Chemistry 2015, 3 (2), 25-29. 
50.Coates, J., Interpretation of Infrared Spectra, A Practical Approach. In Encyclopedia of Analytical Chemistry, Meyers, R. A.; McKelvy, M. L., Eds. Wiley Online Library: 2006.

51.Turick, C. E.; Tisa, L. S.; Caccavo, F., Jr., Melanin production and use as a soluble electron shuttle for Fe(III) oxide reduction and as a terminal electron acceptor by Shewanella algae BrY. Appl Environ Microbiol 2002, 68 (5), 2436-44.

52.Gustavsson, M.; Hornstrom, D.; Lundh, S.; Belotserkovsky, J.; Larsson, G., Biocatalysis on the surface of Escherichia coli: melanin pigmentation of the cell exterior. Sci Rep 2016, 6, 36117.

53.David, C.; Daro, A.; Szalai, E.; Atarhouch, T.; Mergeay, M., Formation of polymeric pigments in the presence of bacteria and comparison with chemical oxidative coupling-II. Catabolism of tyrosine and hydroxyphenylacetic acid by Alcaligenes eutrophus CH34 and mutants. European Polymer Journal 1996, 32 (6), 669-679.

54.Kolczyńska-Szafraniec, U.; Bilińska, B., Infrared studies of natural pheomelanins. Current Topics in Biophysics 1992, 16 (2), 77-80.

55.Wei, W.; Petrone, L.; Tan, Y.; Cai, H.; Israelachvili, J. N.; Miserez, A.; Waite, J. H., An Underwater SurfaceDrying Peptide Inspired by a Mussel Adhesive Protein. Adv Funct Mater 2016, 26 (20), 3496-3507.

56.Xiao, M.; Chen, W.; Li, W.; Zhao, J.; Hong, Y. L.; Nishiyama, Y.; Miyoshi, T.; Shawkey, M. D.; Dhinojwala, A., Elucidation of the hierarchical structure of natural eumelanins. $J R$ Soc Interface 2018, 15 (140).

57.Siwicka, Z. E.; Son, F. A.; Battistella, C.; Moore, M. H.; Korpanty, J.; McCallum, N. C.; Wang, Z.; Johnson, B. J.; Farha, O. K.; Gianneschi, N. C., Synthetic Porous Melanin. J Am Chem Soc 2021, 143 (8), 3094-3103. 58.Dreyer, D. R.; Miller, D. J.; Freeman, B. D.; Paul, D. R.; Bielawski, C. W., Elucidating the Structure of Poly(dopamine). Langmuir 2012, 28 (15), 6428-6435.

59.Di Capua, R.; Gargiulo, V.; Alfè, M.; De Luca, G. M.; Skála, T.; Mali, G.; Pezzella, A., Eumelanin GrapheneLike Integration: The Impact on Physical Properties and Electrical Conductivity. Frontiers in Chemistry 2019, 7 (121).

60.Liu, Q.; Xiao, J.; Liu, B.; Zhuang, Y.; Sun, L., Study on the Preparation and Chemical Structure Characterization of Melanin from Boletus griseus. Int J Mol Sci 2018, 19 (12).

61.Engelen, M.; Vanna, R.; Bellei, C.; Zucca, F. A.; Wakamatsu, K.; Monzani, E.; Ito, S.; Casella, L.; Zecca, L., Neuromelanins of human brain have soluble and insoluble components with dolichols attached to the melanic structure. PLoS One 2012, 7 (11), e48490.

62.Greco, G.; Panzella, L.; Verotta, L.; d'Ischia, M.; Napolitano, A., Uncovering the structure of human red hair pheomelanin: benzothiazolylthiazinodihydroisoquinolines as key building blocks. J Nat Prod 2011, 74 (4), 675-82. 